\title{
Limited granulomatosis with polyangiitis in an adolescent with Crohn's disease on infliximab therapy: cause or coincidence?
}

\author{
Harbir Arora', Shailender Madani², Larisa V. Debelenko³, Eric J. McGrath', Ramakrishna Mutyala ${ }^{4}$ \\ and Lokesh Guglani ${ }^{5}$ \\ 1 Division of Pediatric Infectious Diseases, Carman and Ann Adams Department of Pediatrics, Children's Hospital of Michigan, Detroit, MI, USA \\ 2 Division of Pediatric Gastroenterology, Carman and Ann Adams Department of Pediatrics, Children's Hospital of Michigan, Detroit, MI, USA \\ 3 Division of Pediatric Pathology, Wayne State University School of Medicine, Children's Hospital of Michigan, Detroit, MI, USA \\ 4 Division of Pediatric Hospitalist Medicine, Carman and Ann Adams Department of Pediatrics, Children's Hospital of Michigan, Detroit, MI, USA \\ 5 Division of Pediatric Pulmonary Medicine, Carman and Ann Adams Department of Pediatrics, Children's Hospital of Michigan, Detroit, MI, USA
}

\section{Abstract}

Pulmonary involvement in Crohn's disease (CD) may precede the development of intestinal inflammation, but in most cases occurs during the course of treatment, either as an extra-intestinal manifestation, because of secondary infections, or as a side effect of the therapy itself. This case highlights the differential diagnosis and work up for multiple pulmonary nodules that developed in a patient with $\mathrm{CD}$ who had been in remission on infliximab therapy. Even though infectious causes, such as Mycobacteria and Fungi, account for majority of these cases, the possibility of non-infectious conditions such as autoimmune disorders should also be considered.

Please cite this paper as: Arora H, Madani S, Debelenko LV, McGrath EJ, Mutyala $\mathrm{R}$, Guglani L. Limited granulomatosis with polyangiitis in an adolescent with Crohn's disease on infliximab therapy: cause or coincidence? Clin Respir J 2015; 9: 506-511. DOI:10.1111/crj.12168.

Conflict of interest The authors have no competing interests to declare for this manuscript. This case has not been published or presented earlier.

Abbreviations:
ANA anti-nuclear antibody
ANCA anti-neutrophil cytoplasmic antibody
BAL bronchoalveolar lavage
CD $\quad$ Crohn's disease
CT computed tomography
GPA granulomatosis with polyangiitis
IL interleukin
IV intravenous
LGPA limited form of granulomatosis with polyangiitis
PCR $\quad$ polymerase chain reaction
TB tuberculosis
TNF $\quad$ tumor necrosis factor

\section{Abbreviations:}

BAL bronchoalveolar lavage

CD Crohn's disease

CT computed tomography

GPA granulomatosis with polyangiitis

interleukin

LGPA limited form of granulomatosis with polyangiitis

$P C R$ polymerase chain reaction

TNF tumor necrosis factor

\section{Key words}

adverse effects - Crohn's disease -

granuloma - inflammatory bowel disease infliximab - lung biopsy - lung disease -

pulmonary nodules - tumor necrosis factor

\section{Correspondence}

Lokesh Guglani, MD, FAAP, Division of Pediatric Pulmonary Medicine, Children's Hospital of Michigan, 3901 Beaubien St, Detroit, 48201 Michigan, USA.

Tel: 313-745-5541

Fax: 313-993-2948

email: Iguglani@med.wayne.edu

Received: 28 January 2014

Revision requested: 05 May 2014

Accepted: 16 May 2014

DOI:10.1111/crj.12168

Authorship and Contributorship HA and EJM were involved in the patient's management for infectious disease work up; and helped in writing and editing the manuscript, respectively. SM has been treating the patient, was involved in the diagnostic work up and management, and edited the manuscript. LVD evaluated the lung and colon biopsies, helped in creating the figures and tables for this manuscript and wrote parts of the Discussion section. RM was involved in inpatient management and coordination of the diagnostic work up of this patient. LG was involved in diagnostic work up, wrote the majority of the manuscript and tables and is the corresponding author.

\section{Introduction}

Crohn's disease $(\mathrm{CD})$ is characterised by chronic inflammation in the gastrointestinal tract, typically involving the terminal ileum and/or perianal region, in a transmural, non-contiguous fashion (1). Treatment decisions are based on localisation/extent of disease activity, and associated complications (2). Chimeric anti-tumor necrosis factor-alpha (TNF- $\alpha$ ) antibodies such as infliximab, adalimumab and certolizumab have 
Table 1. Differential diagnosis of granulomatous disorders of the lungs

\begin{tabular}{lc}
\hline Infectious & Non-infectious \\
\hline Mycobacteria & Rheumatologic \\
Mycobacterium & Sarcoidosis \\
tuberculosis & Granulomatosis with polyangiitis \\
Non-tuberculous & (formerly Wegener's \\
mycobacteria & Granulomatosis) \\
& Rheumatoid nodules \\
& Churg Strauss Syndrome \\
Fungi & Hypersensitivity \\
Histoplasma & Hypersensitivity pneumonitis \\
Coccidioides & Talc Granulomatosis \\
Blastomyces & Berylliosis \\
Paracoccidioides & \\
Cryptococcus & \\
Aspergillus & \\
Pneumocystis & \\
Parasitic infestations & Immune \\
Cestodes & Chronic granulomatous disease \\
Echinococcus & Granulomatous Lymphocytic \\
Nematodes & Interstitial Lung Disease \\
Toxocara & (associated with Common \\
Dirofilaria & Variable Immune Deficiency) \\
Trematodes & Lymphocytic Interstitial Pneumonitis \\
Schistosoma & in HIV infected individuals \\
Paragonimus & \\
\hline
\end{tabular}

been used to induce remission, either alone or in combination with other agents (3).

Pulmonary involvement in $\mathrm{CD}$ can sometimes precede the development of intestinal inflammation (4), or occur during the course of treatment - either as an extra-intestinal manifestation of CD (5), because of secondary infections or as a side effect of the therapy itself (6). Granulomatous inflammation within the airways or lung parenchyma can occur due to CD but can also occur because of several other forms of primary and secondary lung disorders (Table 1). We present an unusual case of a 19-year old patient with stable CD who developed multiple pulmonary nodules while on therapy with infliximab. The approach to initial diagnostic work up, differential diagnosis of granulomatous lung disorders and their salient differentiating features on lung histology, as well as management is discussed.

\section{Case report}

A 19-year old Caucasian female with history of CD was admitted with sudden onset of severe, sharp chest pain, localised to the left upper sternal border, radiating to the left neck and shoulder; worsening with deep inspiration. There was no history of trauma to the chest, shortness of breath, palpitations, sweating, fever, cough or hemoptysis. CD with perianal fistulae was diagnosed 5 years ago and well controlled with intravenous infliximab infusion given every 7 weeks. On examination, she had stable vital signs with shallow breaths but normal oxygen saturation on room air. Eczematous dry skin was noted on her feet. The rest of the examination was benign. Initial laboratory results revealed white blood cell count of $11000 / \mathrm{mm}^{3}$ with normal differential, hemoglobin $13.3 \mathrm{gm} / \mathrm{dL}$, hematocrit of $39.1 \%$, platelets $355000 / \mathrm{mm}^{3}$; C-reactive protein, $132 \mathrm{mg} / \mathrm{L}$, erythrocyte sedimentation rate, $49 \mathrm{~mm}$, D-dimer $0.5 \mathrm{mg} / \mathrm{L}$ (normal < 0.59). Initial chest radiograph was normal (Fig. 1A). Because of oral contraceptive use (ethinyl estradiol/norgestimate), a chest computed tomography (CT) scan done to rule out pulmonary embolism revealed the presence of multiple pulmonary nodules, with the largest one in left lower lobe measuring $1.3 \mathrm{~cm}$ (Fig. 1B).

Given the patient's infliximab use, an infectious aetiology was initially suspected and further treatment with infliximab withheld. Blood for bacterial culture, fungal serology and fungal antigens (beta-d-glucan, cryptococcal and blastomyces antigen) were negative. A flexible fibre-optic bronchoscopy showed normal airway anatomy and mucosal surfaces. Bacterial, mycobacterial and fungal stains and culture; mycoplasma and viral cultures; pneumocystis stain and polymerase chain reaction (PCR) for viral DNA from bronchoalveolar lavage (BAL) fluid were all negative. Negative purified protein derivative skin test and serum interferon gamma release assay ruled out mycobacterial infections.

In the next few days there was worsening of chest pain with tachypnea and a repeat chest CT showed increase in the number and size of the pulmonary nodules (Fig. 1C), and CT abdomen was negative for mass lesions. Intravenous amphotericin B and ceftriaxone were started empirically. Further investigations revealed serum anti-nuclear antibody titre of 1:640, negative anti-dsDNA, c-anti-neutrophil cytoplasmic antibody (c-ANCA), p-ANCA and anti-infliximab antibodies; and normal levels of complement (C3 and $\mathrm{C} 4$ ), angiotensin-converting enzyme, serum calcium and Vitamin D. A negative nitro blue tetrazolium test ruled out chronic granulomatous disease, and bone marrow biopsy was negative for hematopoietic malignancy. Finally, an open lung biopsy was obtained for evaluation of lung pathology and microbiology. The special stains of lung tissue were negative for fungus 
Figure 1. Radiologic Images for index case. (A) Chest radiograph (anteroposterior view) at the time of initial presentation. (B) Chest computed tomography (CT) scan at initial presentation showing multiple small subpleural nodules. (C) Chest CT scan 1 week later showing increased size and number of lung nodules. (D) Chest CT scan 3 months after initiation of treatment showing near complete resolution of nodules.
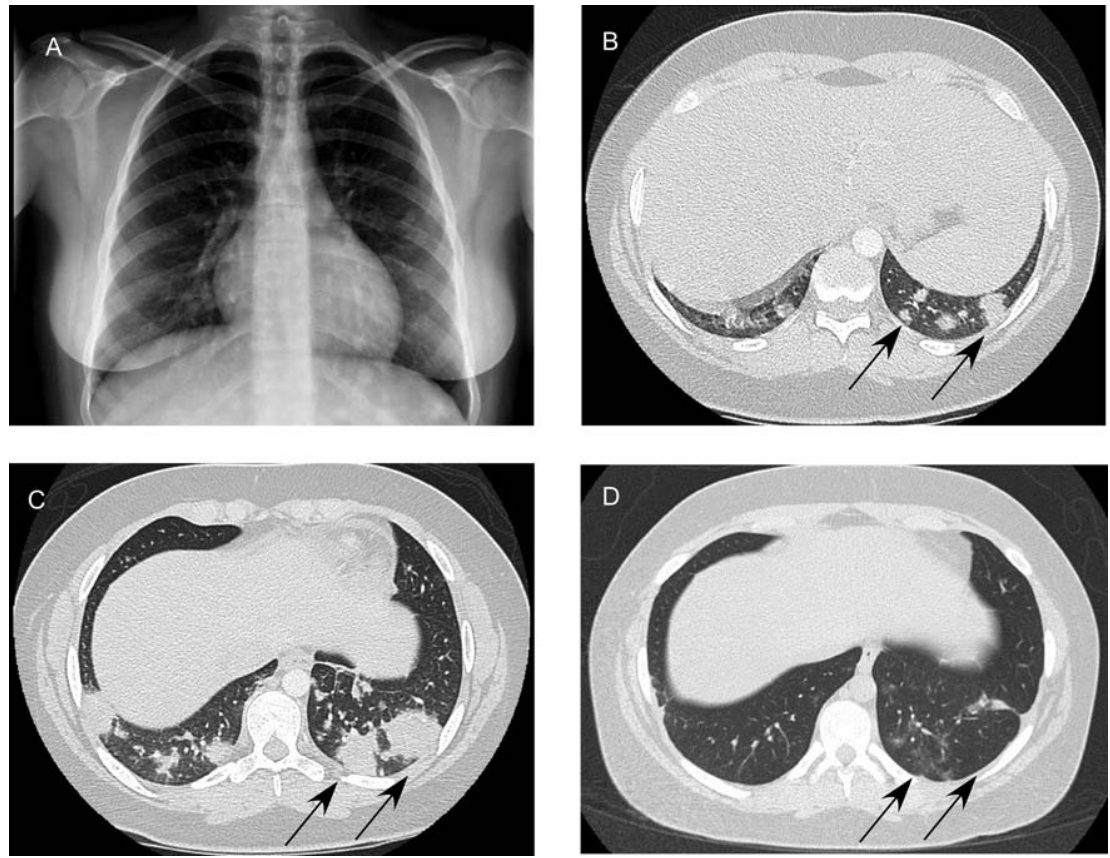

and mycobacteria, including pan-bacterial, -fungal and -mycobacterial PCR testing.

Histopathology of lung nodules revealed massive geographic necrosis and necrotising granulomas that were surrounded by palisading histiocytes (Fig. 2A). Pulmonary vasculitis with adventitial fibrosis, focal intimal hyperplasia and sub-endothelial fibrohistiocystic proliferation with presence of fibrin thrombi in smaller vessels were also noted (Fig. 2B). Inflammatory changes were seen in bronchioles, pleura and alveoli. The observed picture of geographic necrosis and palisading granulomas with neutrophilic/ karyorrhectic centres as well as vasculitis, in the absence of infection, was consistent with limited form of granulomatosis with polyangiitis (LGPA) (Table 2). The diagnosis of LGPA was confirmed by an additional independent review by three pulmonary pathologists from another academic institution. Lower gastrointestinal biopsies performed a month later to assess CD activity revealed minimally active $\mathrm{CD}$ with rare small non-necrotising epitheloid granulomas (Fig. 2C) and focal cryptitis (Fig. 2D).

Treatment with high-dose intravenous steroids for 3 days followed by a tapered regimen was instituted. The patient showed rapid clinical improvement in her symptoms within hours of initiation of the steroid therapy. She was also started on methotrexate $20 \mathrm{mg}$ subcutaneous once a week (as additional steroidsparing therapy). Follow-up chest CT scan done
3 months later (Fig. 1D) showed complete resolution of the nodules.

\section{Discussion}

The differential diagnosis of granulomatous lung disorders includes various infectious and non-infectious causes (Table 1) (7). Further delineation could be done based on specific features of the granulomas and other cellular components (Table 2). This case highlights the important differential diagnoses for pulmonary lesions in a patient with $\mathrm{CD}$ who had been in remission on infliximab therapy. The initial concern was for an infectious aetiology which is generally the most common cause (8), but it was ruled out in our case after negative special stains and cultures of BAL and lung tissue for fungal and mycobacterial organisms; and negative advanced PCR testing results. The lung biopsy showed necrotising granulomatous inflammation with angiocentric granulomas and vasculitis, and this narrowed our differential diagnosis to pulmonary involvement in $\mathrm{CD}$, granulomatosis with polyangiitis (GPA) and sarcoidosis (Table 2). However, our patient had been asymptomatic from the standpoint of gastrointestinal symptoms, had a normal magnetic resonance enterography study, with minimal activity on lower gastrointestinal biopsy. Geographic necrosis and vasculitis are generally lacking within the granulomas seen with pulmonary involvement in CD (9), whereas 

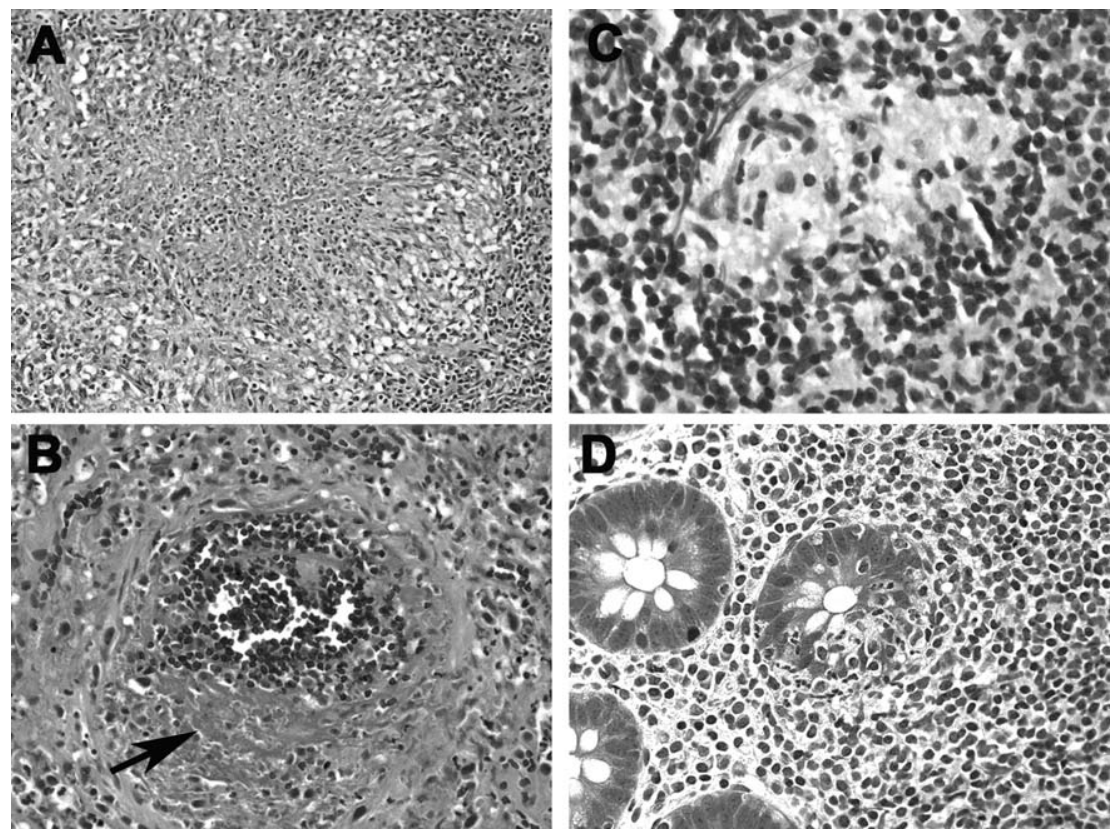

Figure 2. Pulmonary and intestinal pathology. (A) Pulmonary biopsy demonstrating a palisading granuloma with necrotic centre filled with neutrophils and karyorrhectic debris and surrounded by histiocytes. Hematoxylin-eosin, original magnification $\times 200$. (B) Pulmonary biopsy demonstrating a small artery involved by vasculitis with fibrin deposits (arrow). Hematoxylin-eosin, original magnification $\times 400$. (C) Colonic biopsy demonstrating a small compact non-necrotising granuloma composed of epitheloid histiocytes, characteristic of Crohn's disease. Hematoxylin-eosin, original magnification $\times 600$. Note the difference between the appearance of colonic (C) and pulmonary (A) granulomas. (D) Colonic biopsy demonstrating chronic, mildly active colitis with a single crypt involved by cryptitis in the centre. Hematoxylin-eosin, original magnification $\times 400$.

Table 2. Distinguishing features of non-infectious granulomatous inflammation of the lungs (pulmonary Crohn's disease, granulomatosis with polyangiitis and sarcoidosis) (10)

\begin{tabular}{|c|c|c|c|}
\hline Features & $\begin{array}{l}\text { Primary Crohn's disease } \\
\text { involving the lung }\end{array}$ & Granulomatosis with polyangiitis & Sarcoidosis \\
\hline Distribution & Bronchocentric & Angiocentric, pleural based & $\begin{array}{l}\text { Diffuse bronchovascular with hilar } \\
\text { adenopathy }\end{array}$ \\
\hline Granulomas & $\begin{array}{l}\text { Small Non-necrotic, } \\
\text { non-caseating granulomas } \\
\text { with epitheloid histiocytes }\end{array}$ & $\begin{array}{l}\text { Necrotising granulomas of various } \\
\text { sizes, frequently large, geographic, } \\
\text { with neutrophilic/karyorrhectic } \\
\text { centres and palisading histiocytes } \\
\text { with occasional multinucleated giant } \\
\text { cells at the periphery }\end{array}$ & $\begin{array}{l}\text { Uniform small-to-medium sized } \\
\text { granulomas, characteristically } \\
\text { non-necrotising composed of } \\
\text { epitheloid histiocytes and frequent } \\
\text { multinucleated giant cells }\end{array}$ \\
\hline $\begin{array}{l}\text { Presence of } \\
\text { necrosis }\end{array}$ & Generally non-necrotising & $\begin{array}{l}\text { Central necrosis may be confluent or } \\
\text { show irregular serpiginous pattern } \\
\text { known as 'geographic necrosis' }\end{array}$ & $\begin{array}{l}\text { Generally non-necrotising; small areas } \\
\text { of central necrosis can be } \\
\text { occasionally seen. }\end{array}$ \\
\hline $\begin{array}{l}\text { Vascular } \\
\text { changes }\end{array}$ & None & $\begin{array}{l}\text { Necrotising vasculitis affecting small to } \\
\text { medium sized vessels }\end{array}$ & None \\
\hline Inclusions & None & None & $\begin{array}{l}\text { Asteroid Bodies } \\
\text { Schaumann bodies } \\
\text { Hamazaki-Wesenberg bodies } \\
\text { Calcium oxalate }\end{array}$ \\
\hline
\end{tabular}


the granulomas seen in sarcoidosis are typically well formed, non-necrotising, with numerous epitheloid cells and giant cells. As seen in our case, the granulomas seen in GPA are generally poorly formed, show palisading histiocytes, and are accompanied by vasculitis and a mixed inflammatory infiltrate (10).

Pulmonary involvement in CD is variable and may result from disease itself or associated medical therapy. Casey et al. reported chronic bronchitis and bronchiolitis with non-necrotising granulomatous inflammation as the most common forms $(9 / 25 ; 36 \%)$ of pulmonary involvement (11). Many of the agents used for therapy of inflammatory bowel diseases (including mesalamine) are known to cause pulmonary side effects (12). Tayer-Shifman et al. reported a case of CD with multiple pulmonary cavitary nodules and c-ANCA positivity that was attributed to mesalamine (13), but their patient had complete resolution of pulmonary nodules after its discontinuation (even though c-ANCA positivity continued). Our patient had not been treated with mesalamine and had negative c-ANCA. This brings up the question of whether there is a link between the pathways of inflammation that are involved in CD and GPA and whether immune modulators (such as mesalamine or infliximab) could induce other alternative immune mechanisms/pathways that increases the risk for the development of autoimmune disease and/or vasculitis in these patients. There is some indication that the interleukin-17 (IL17) pathway could be the common link in these two conditions (14).

Infliximab has been used for therapy of $\mathrm{CD}$ for more than a decade now, and a review of 233 cases treated with anti-TNF alpha therapies showed that cutaneous vasculitis was the most common manifestation, and lung involvement primarily occurred in the form of interstitial lung disease (15). There have been several reports of infectious (16-18) and non-infectious (6, 19-21) pulmonary complications in patients with CD while on infliximab therapy. Hypersensitivity vasculitis with leukocytoclastic vasculitis $(22,23)$ due to use of infliximab was unlikely because of lack of cutaneous involvement in our patient.

There is a possibility of secondary autoimmune disease developing in patients on long-term anti-TNF therapy, and a recent review by Ramos-Casals (15) reported that one quarter of these patients had extracutaneous vasculitis. To our knowledge, there are only two other case reports that have reported the occurrence of GPA in patients with $\mathrm{CD}$, but none of those patients had been on infliximab therapy $(24,25)$. Our patient's presentation fits with the description of 'limited' form of GPA, which is known to be localised to the lungs, is more common in females, has a younger age of onset, and is less likely to be associated with ANCA positivity (26). ANCA negative GPA is generally associated with lower incidence of renal involvement and better long-term survival (27). ANCA positivity is not an absolute requirement for the diagnosis of GPA as per the American College of Rheumatology criteria (28) or in the updated definition provided by the Chapel Hill Consensus Conference (29).

To conclude, our case highlights the fact that secondary autoimmune disease can develop in patients with $\mathrm{CD}$ on infliximab therapy and that nodular pulmonary lesions having features of granulomatous inflammation have a broad differential diagnosis. We have also highlighted the importance of lung histopathology in establishing the diagnosis. Even though infectious causes account for majority of these cases, the possibility of non-infectious conditions such as autoimmune disorders should be considered after carefully excluding infectious organisms such as mycobacteria and fungi.

\section{Acknowledgements}

The authors would like to acknowledge the help of Drs Jeffrey L. Myers, Amir Lagstein and Lindsay Schmidt from Department of Pathology, University of Michigan Health System for their input on interpretation of lung pathology for this case.

\section{References}

1. Khor B, Gardet A, Xavier RJ. Genetics and pathogenesis of inflammatory bowel disease. Nature. 2011;474(7351): 307-17.

2. Baran B, Karaca C. Practical medical management of Crohn's disease. ISRN Gastroenterol. 2013;2013: 208073; doi: http://dx.doi.org/10.1155/2013/208073.

3. Feagan BG, McDonald JW, Panaccione R, et al. Methotrexate in combination with infliximab is no more effective than infliximab alone in patients with Crohn's disease. Gastroenterology. 2014;146: 681-88 e1.

4. Levenbrown Y, Tauber D, Hall OR, Baldridge AD. Granulomatous lung disease as the initial presentation of Crohn's disease. J Pediatr Gastroenterol Nutr. 2009;48(4): 487-90.

5. Krishnan S, Banquet A, Newman L, Katta U, Patil A, Dozor AJ. Lung lesions in children with Crohn's disease presenting as nonresolving pneumonias and response to infliximab therapy. Pediatrics. 2006;117(4): 1440-3.

6. Panagi S, Palka W, Korelitz BI, Taskin M, Lessnau KD. Diffuse alveolar hemorrhage after infliximab treatment of Crohn's disease. Inflamm Bowel Dis. 2004;10(3): 274-7.

7. Mukhopadhyay S, Gal AA. Granulomatous lung disease: an approach to the differential diagnosis. Arch Pathol Lab Med. 2010;134(5): 667-90. 
8. Mukhopadhyay S. Role of histology in the diagnosis of infectious causes of granulomatous lung disease. Curr Opin Pulm Med. 2011;17(3): 189-96.

9. Puntis JW, Tarlow MJ, Raafat F, Booth IW. Crohn's disease of the lung. Arch Dis Child. 1990;65(11): 1270-1.

10. Tomasefski JF, Cagle PT, Farver CF, Fraire AE. Dail and Hammar's Pulmonary Pathology Volume 1: Non Neoplastic Lung Disease. New York, Springer, 2008.

11. Casey MB, Tazelaar HD, Myers JL, Hunninghake GW, Kakar S, Kalra SX, Ashton R, Colby TV. Noninfectious lung pathology in patients with Crohn's disease. Am J Surg Pathol. 2003;27(2): 213-19.

12. Alskaf E, Aljoudeh A, Edenborough F. Mesalazineinduced lung fibrosis. BMJ Case Rep. 2013; doi: 10.1136/bcr-2013-008724.

13. Tayer-Shifman OE, Shuvy M, Hershko AY. Mesalamine-induced multiple pulmonary cavitary nodules associated with cytoplasmic antineutrophil cytoplasmic antibody (c-ANCA). J Clin Rheumatol. 2009;15(5): 256-7.

14. Muller A, Lamprecht P. [Interleukin-17 in chronic inflammatory and autoimmune diseases: rheumatoid arthritis, Crohn's disease and Wegener's granulomatosis]. Z Rheumatol. 2008;67(1): 72-4.

15. Ramos-Casals M, Brito-Zeron P, Munoz S, Soria N, Galiana D, Bertolaccini L, Cuadrado MJ, Khamashta MA. Autoimmune diseases induced by TNF-targeted therapies: analysis of 233 cases. Medicine (Baltimore). 2007;86(4): 242-51.

16. Lim LT, Ruzmetova N, Ballinger SH, Moorthy RS. Acute pulmonary histoplasmosis in a patient with uveitis after infliximab therapy. Int Ophthalmol. 2011;31(4): 349-51.

17. Warris A, Bjorneklett A, Gaustad P. Invasive pulmonary aspergillosis associated with infliximab therapy. N Engl J Med. 2001;344(14): 1099-100.

18. Hirai F, Matsui T, Ishibashi Y, Higashi D, Futami K, Haraoka S, Iwashita A. Asymptomatic pulmonary cryptococcosis in a patient with Crohn's disease on infliximab: case report. Inflamm Bowel Dis. 2011;17(7): 1637-8.

19. Heraganahally SS, Au V, Kondru S, Edwards S, Bowden JJ, Sajkov D. Pulmonary toxicity associated with infliximab therapy for ulcerative colitis. Intern Med J. 2009;39(9): 629-30.

20. Caccaro R, Savarino E, D'Inca R, Sturniolo GC. Noninfectious interstitial lung disease during infliximab therapy: case report and literature review. World J Gastroenterol. 2013;19(32): 5377-80.

21. Sen S, Peltz C, Jordan K, Boes TJ. Infliximab-induced nonspecific interstitial pneumonia. Am J Med Sci. 2012;344(1): 75-8.

22. McIlwain L, Carter JD, Bin-Sagheer S, Vasey FB, Nord J. Hypersensitivity vasculitis with leukocytoclastic vasculitis secondary to infliximab. J Clin Gastroenterol. 2003;36(5): 411-13.

23. Karoui S, Bibani N, Ben Gorbel I, Serghini M, Mlika M, Braham A, Chelly I, Haouet S, Houmene H, Filali A. Leukocytoclastic vasculitis: a rare adverse effect secondary to infliximab. Inflamm Bowel Dis. 2011;17(2): E4-5.

24. Sieczkowska A, Lewandowski P, Szumera M, Kaminska B. [Coexistence of Crohn disease and Wegener granulomatosis in a 15-year-old patient]. Med Wieku Rozwoj. 2011;15(4): 472-6.

25. Codish S, Abu-Shakra M, Depsames R, Sion-Vardy N, Benharroch D, Sukenik S. Wegener's granulomatosis in a patient with Crohn's disease. Isr Med Assoc J. 2000;2(8): 630-1.

26. Stone JH. Wegener's Granulomatosis Etanercept Trial Research G. Limited versus severe Wegener's granulomatosis: baseline data on patients in the Wegener's granulomatosis etanercept trial. Arthritis Rheum. 2003;48(8): 2299-309.

27. Kim HW, Kim JW, Im CH, Shin KC, Lee EY, Lee EB, Song YW. The clinicopathologic characteristics of granulomatosis with polyangiitis (Wegener's): a retrospective study of 45 patients in Korea. Mod Rheumatol. 2013;23(5): 864-71.

28. Leavitt RY, Fauci AS, Bloch DA, et al. The American College of Rheumatology 1990 criteria for the classification of Wegener's granulomatosis. Arthritis Rheum. 1990;33(8): 1101-7.

29. Jennette JC, Falk RJ, Andrassy K, et al. Nomenclature of systemic vasculitides. Proposal of an international consensus conference. Arthritis Rheum. 1994;37(2): 187-92. 